\title{
Resistin-like molecule beta augments phenotypic modulation of human aortic smooth muscle cell triggered by high glucose
}

\author{
Yi Wang ${ }^{1)}$, Yawen Zhang ${ }^{1)}$, Xiangyu Gao ${ }^{1)}$, Jiali Qian ${ }^{1)}$, Jia Yang ${ }^{1)}$, Wanwan Sun ${ }^{1)}$, Hao Wang ${ }^{2)}$ and \\ Yehong Yang ${ }^{1)}$
}

\author{
1) Department of Endocrinology, Huashan Hospital, Fudan University, Shanghai 200040, China \\ 2) Shanghai Medical College, Fudan University, Shanghai 200040, China
}

\begin{abstract}
Vascular muscle cells (VSMCs) participate in the pathophysiology of atherosclerosis. Resistin-like molecule beta $(\operatorname{Re} \operatorname{lm} \beta)$ contributes to atherosclerosis development by activating macrophage. This study aims to investigate whether Relm $\beta$ regulates VSMC phenotypic modulation under high glucose environment. Human aortic vascular smooth muscle cells were cultured and treated with Relm $\beta$ in the presence or absence of high glucose. VSMC phenotypic modulation was assessed by expression of related markers. The migration of VSMCs was detected by wound healing assay and transwell assay. The proliferation of VSMCs was measured using CCK- 8 assay. In this study, we observed that Relm $\beta$ modulated VSMC phenotypic modulation by down-regulating expression of smooth muscle $\alpha$-actin ( $\alpha$-SMA), smooth muscle myosin heavy chain (SM-MHC), and calponin while up-regulating expression of osteopontin (OPN). Relm $\beta$ increased the expression of inflammatory genes in VSMCs. Relm $\beta$ also augmented VSMCs migration as well as proliferation. It is worth noting that all the effects of VSMCs were enhanced upon high glucose stimulation. The phosphorylation levels of p38MAPK and ERK1/2 were increased by co-treatment with Relm $\beta$ and high glucose. The p38 MAPK pathway inhibitor RWJ64809 and pERK1/2 inhibitor PD98059 significantly inhibited the proliferation of VSMCs induced by Relm $\beta$ and high glucose. Our results provide evidence that $\operatorname{Re} \operatorname{lm} \beta$ augments phenotypic modulation and migration of human aortic smooth muscle cell induced by high glucose. Relm $\beta$ might be a potential target for treatment of atherosclerosis induced by hyperglycemia.
\end{abstract}

Key words: Resistin-like molecule beta, Vascular smooth muscle cell, Phenotypic modulation, High glucose

\begin{abstract}
ATHEROSCLEROSIS is a key cause of various vascular diseases and has high morbidity and mortality worldwide. Hyperglycemic status activates multiple maladaptive signaling pathways and leads to the occurrence and progression of atherosclerotic diseases with distinct characteristics [1]. Macrophage accumulation in the vascular wall is a hallmark of atherosclerosis [2]. Recently, accumulating studies reveal that vascular smooth muscle cells

Submitted Jun. 21, 2020; Accepted Nov. 16, 2020 as EJ20-0343 Released online in J-STAGE as advance publication Jan. 13, 2021 Correspondence to: Yehong Yang, MD, Department of Endocrinology, Huashan Hospital, Fudan University, 12 Middle Urumqi Road, Shanghai 200040, China.

E-mail: yehongyang@fudan.edu.cn

Abbreviations: VSMCs, vascular smooth muscle cells; HASMC, Human aortic vascular smooth muscle cells; Relm $\beta$, resistin-like molecule beta; $\alpha$-SMA, smooth muscle $\alpha$-actin; MHC, smooth muscle myosin heavy chain; OPN, osteopontin; MMP-2, matrix metalloproteinase-2; MMP-9, matrix metalloproteinase-9; PDGF, platelet-derived growth factor; PDGFR, platelet-derived growth factor receptor; CDK, cyclin-dependent kinase; MAPKs, mitogenactivated protein kinases.
\end{abstract}

(VSMCs) and especially VSMC phenotypic modulation play an important role in the development of atherosclerosis. VSMCs possess splendid phenotypic plasticity, which allows rapid adaptation upon various environmental stimuli. Upon vascular damage, contractile VSMCs will dedifferentiate into a synthetic, proliferative and migratory phenotype (known as phenotypic modulation), which contributes to vascular remodeling during atherosclerosis [3, 4]. However, little is known about how the VSMC phenotypic modulation is regulated, especially under hyperglycemic status.

Resistin and the resistin-like molecules (Relms) comprise a novel class of cysteine-rich proteins, which include resistin, Relm $\alpha, \operatorname{Re} \operatorname{lm} \beta$ and Relm $\gamma$. Resistin and $\operatorname{Re} \operatorname{lm} \beta$, but not Relm $\alpha$ and $\operatorname{Re} \ln \gamma$, are expressed in humans $[5,6]$. Resistin is initially identified as an adipokine, which can reduce insulin action. $\operatorname{Re} \operatorname{lm} \beta$ is produced limitedly in the colon and the lung; and it is report to be involved in local immune response regulation in the gut and bronchial epithelial cells $[7,8]$. Relm $\beta$ produced by colon goblet cells is induced during intestinal 
inflammation, including microbiota and high-fed diet [5, $9,10]$. Relm $\beta$ also contributes to hypoxia-induced pulmonary vascular remodeling [6], pathogenesis of colon cancer [11] and type 2 diabetes [12].

Recently, Relm $\beta$ is identified in activated macrophages and demonstrated to accelerate the development of atherosclerosis [13]. $\operatorname{Re} \operatorname{lm} \beta$ is found to be expressed in the foam cells of atherosclerotic lesion in both human samples and ApoE-/- mice. Relm $\beta$ activates the classical M1 macrophage and contributes to the atherosclerosis development and plaque instability [13]. However, whether $\operatorname{Relm} \beta$ is involved in the regulation of VSMCs, which also play an important role in atherosclerosis development, is unknown.

In this study, we showed that Relm $\beta$ modulated the behaviors of VSMCs, including phenotypic modulation, inflammation, migration and proliferation, after high glucose stimulation. We also found that mitogenactivated protein kinases (MAPKs) signaling pathway participated in VSMC phenotypic proliferation induced by co-stimulation of Relm $\beta$ and high glucose.

\section{Material and Methods}

\section{Cell culture and treatment}

Human aortic vascular smooth muscle cells (HASMCs) were purchased from Sciencell. They were cultured in Smooth Muscle Cell Medium (SMCM) (Sciencell, US). The cells of the third to the eighth-generation were used for the following experiments. The $25 \mathrm{mM}$ high glucose medium was used for high glucose intervention. The $20 \mathrm{ng} / \mathrm{mL}$ Relm $\beta$ (ProSpec, Israel) was used for Relm $\beta$ treatment. For Relm $\beta$ and high glucose co-treatment, the cells were stimulated by $20 \mathrm{ng} / \mathrm{mL}$ RELM and $25 \mathrm{mM}$ high glucose simultaneously.

\section{Western blot analysis}

Cells were lysed with Radio-Immunoprecipitation Assay solution (Thermo, US) containing the protease inhibitor on ice for $20 \mathrm{~min}$ with rotation. Lysate was collected and the supernatant was collected after centrifugation for $30 \mathrm{~min}$ at $4^{\circ} \mathrm{C}$. Protein concentration was determined using BCA protein assay kit (Beyotime, China). Proteins $(20 \mu \mathrm{g})$ were seperated on $10 \%$ SDSPAGE gels and transferred to PVDF membranes. The membranes were blocked by $5 \%$ milk for 1 hour, and then incubated with targeted protein primary antibody overnight. The membranes were incubated with horseradish peroxidase-conjugated secondary antibody (Boster, China). The blots were developed with enhanced chemiluminescence detection reagents.

\section{RT-PCR}

Total RNA was extracted with TRIzol (Thermo, US) according to the manufacturer's instructions. RNA was reverse transcribed into cDNA using M-MLV (Takara, Japan). Real-time PCR (RT-PCR) was performed using SYBR Green Mix (Applied Biosystems, Woolston Warrington, UK). The total volume for RT-PCR was $20 \mu \mathrm{L}$. The PCR procedures were as follows: denaturation at $50^{\circ} \mathrm{C}$ for $2 \mathrm{~min}$, annealing at $60^{\circ} \mathrm{C}$ for 30 seconds and extension at $72^{\circ} \mathrm{C}$ for 60 seconds with a total of 40 cycles. The results were analyzed with $2^{-\Delta \Delta \mathrm{Ct}}$ method.

\section{Wound healing assay}

HASMCs were seeded in the six-well plates and cultured with serum-free SMCM for 24 hours. A $200 \mu \mathrm{L}$ of pipettor was used to streak the bottom of each well and PBS was used to wash away the suspended cells. Then complete medium was added into the culture plate. Pictures were taken under the microscope and digital images were obtained every 24 hours.

\section{Transwell assay}

HASMCs were starved with serum-free culture medium for 24 hours. Then, five thousand HASMCs were seeded in each transwell chamber (Corning, US). The $200 \mu \mathrm{L}$ serum-free medium was added into the lower chamber and $500 \mu \mathrm{L}$ SMCM medium containing $10 \%$ FBS was added into the upper chamber. SMCM medium containing $10 \%$ FBS was removed from the upper chamber after 24 hours of culture. The cells were gently washed with PBS and fixed with $4 \%$ paraformaldehyde for $30 \mathrm{~min}$. Image were observed and taken under a light microscope.

\section{CCK-8 assay}

HASMCs were seeded in 96-well plates as a density of 5,000 cells per well and treated according to the indicated treatments and timepoints. The $10 \mu \mathrm{L}$ of CCK8 solution was added to each well followed by incubation for 30 minutes at $37^{\circ} \mathrm{C}$. Absorbance value at $450 \mathrm{~nm}$ was measured by a microplate reader.

\section{Statistical analysis}

Data are expressed as mean \pm standard difference (SD). Statistical analysis was performed using SPSS 25.0 (IBM). The $t$-test was used for comparison between groups. Statistical significance was indicated at $* p<$ $0.05, * * p<0.01$, and $* * * p<0.001$. Graphs was plotted using GraphPad Prism 8. 


\section{Results}

\section{Relm $\beta$ promotes the phenotypic modulation and inflammation of VSMCs induced by high glucose}

To investigate how Relm $\beta$ modulates the phenotype of VSMCs, we treated the cells with high glucose, $\operatorname{Re} \operatorname{lm} \beta$ and the combination of high glucose and $\operatorname{Re} \operatorname{lm} \beta$ and assessed specific markers of contractile VSMCs, including smooth muscle $\alpha$-actin ( $\alpha$-SMA), smooth muscle myosin heavy chain (MHC), calponin, matricellular protein, and osteopontin (OPN). As shown in Fig. 1A, $\operatorname{Re} \operatorname{lm} \beta$ as well as high glucose significantly regulated the expression of phenotypic modulation-related genes. They suppressed the expression of $\alpha$-SMA, SM-MHC and calponin, but elevated the expression of OPN. It is worthy to note that $\operatorname{Re} \operatorname{lm} \beta$ treatment could further downregulate the expression of $\alpha$-SMA, SM-MHC and calponin and upregulate the expression of OPN upon high glucose stimulation. Consistent with gene expression results, $\alpha-$ SMA protein expression was also reduced by high glucose. Although Relm $\beta$ alone didn't affect $\alpha$-SMA protein content significantly, Relm $\beta$ suppressed $\alpha$-SMA protein level in the presence of high glucose (Fig. 1B). Meanwhile, high glucose or Relm $\beta$ treatment induced the expression of OPN (Fig. 1A). Relm $\beta$ treatment further enhanced the expression of OPN upon high glucose stimulation (Fig. 1B). Collectively, these data demonstrate that $\operatorname{Re} \operatorname{lm} \beta$ promotes the phenotypic modulation of VSMCs induced by high glucose.

It is well-known that atherosclerosis is an inflammatory disease and immune response is involved in atherosclerosis development [4, 14]. We therefore assessed whether Relm $\beta$ treatment affects the inflammatory gene expression. we found that high glucose significantly upregulated the expression of IL-1 $\beta$, IL-8, TGF- $\beta$, $\mathrm{TNF} \alpha$, and MCP-1, which are classic inflammatory markers. Relm $\beta$ treatment augmented the upregulation of these inflammatory markers induced by high glucose, while $\operatorname{Re} \operatorname{lm} \beta$ alone didn't increase the expression of inflammatory genes (except MCP-1) dramatically (Fig. 1C). These data suggest that $\operatorname{Re} \operatorname{lm} \beta$ increases inflammatory gene expression in VSMCs upon high glucose treatment.

\section{Relm $\beta$ induces VSMCs migration upon high glucose stimulation}

Wound healing assay was used to demonstrate the effect of Relm $\beta$ and high glucose on VSMCs migration. Compared with non-treated cells, $20 \mathrm{ng} / \mathrm{mL}$ Relm $\beta$ treatment, as well as high glucose, remarkably enhanced cell migration (Fig. 2A). In line with the phenotypic modulation, Relm $\beta$ and high glucose synergistically enhanced the cell migration. Next, the transwell Boyden chamber experiment was performed in order to confirm the promoting effect of Relm $\beta$ on VSMCs migration. As expected, the number of VSMCs that migrated through the transwell chamber was strikingly induced by treatment with high glucose for $24 \mathrm{~h}$ or Relm $\beta$ for $24 \mathrm{~h}$. Noteworthy, VSMCs migration was further enhanced when cells were co-treated with Relm $\beta$ and high glucose (Fig. 2B). Furthermore, we investigated the regulatory effects of Relm $\beta$ on the migration markers. As shown in Fig. $2 \mathrm{C}$, migration markers, including matrix metalloproteinase-2 (MMP-2) and matrix metalloproteinase-9 (MMP-9), were significantly higher in high glucose or $\operatorname{Re} \operatorname{lm} \beta$ treated cells compared with control cells. Relm $\beta$ treatment further elevated MMP-2 and MMP-9 gene expression upon high glucose treatment. Platelet-derived growth factor-platelet-derived growth factor receptor (PDGF-PDGFR) signaling represents one of the most prominent inducers of VSMC migration and proliferation. VSMCs can secrete PDGF in response to some stimulation and accelerate atherosclerosis [15]. In our study, consistent results were observed in expression of PDGFA and PDGFR-A after Relm $\beta$ and high glucose treatment (Fig. 2C). These findings suggest that Relm $\beta$ induces VSMCs migration upon high glucose stimulation.

\section{Relm $\beta$ stimulates proliferation of VSMCs upon high glucose treatment}

To explore whether Relm $\beta$ affects the proliferation of VSMCs with or without high glucose, we performed CCK-8 assay after treating VSMCs with Relm $\beta$ with or without high glucose. As shown in Fig. 3A, the proliferation of VSMCs was significantly induced by $\operatorname{Re} \operatorname{lm} \beta$ or high glucose treatment, as well as Relm $\beta$ and high glucose co-treatment. To further confirm the effect of Relm $\beta$ on proliferation, we tested the expression of cell phasespecific cyclin-dependent kinase (CDK)-cyclins. The results showed that the protein levels of CDK2, cyclin D1, CDK4, and cyclin E were greatly increased by high glucose stimulation. Relm $\beta$ slightly increased the expression of CDK2, cyclin D1, CDK4, and cyclin E. However, this effect was tremendously augmented by cotreatment with high glucose (Fig. 3B, 3C). This result suggests that Relm $\beta$ stimulates proliferation of VSMCs upon high glucose treatment.

\section{MAPKs signaling pathway maybe involved in proliferation of VSMC induced by Relm $\beta$ and high glucose co-stimulation}

It is reported that MAPKs signaling pathway, especially ERK1/2 signaling pathway, is a major driver of VSMC proliferation and inflammation [16]. Relm $\beta$ also regulates proliferation of human diabetic nephropathy 

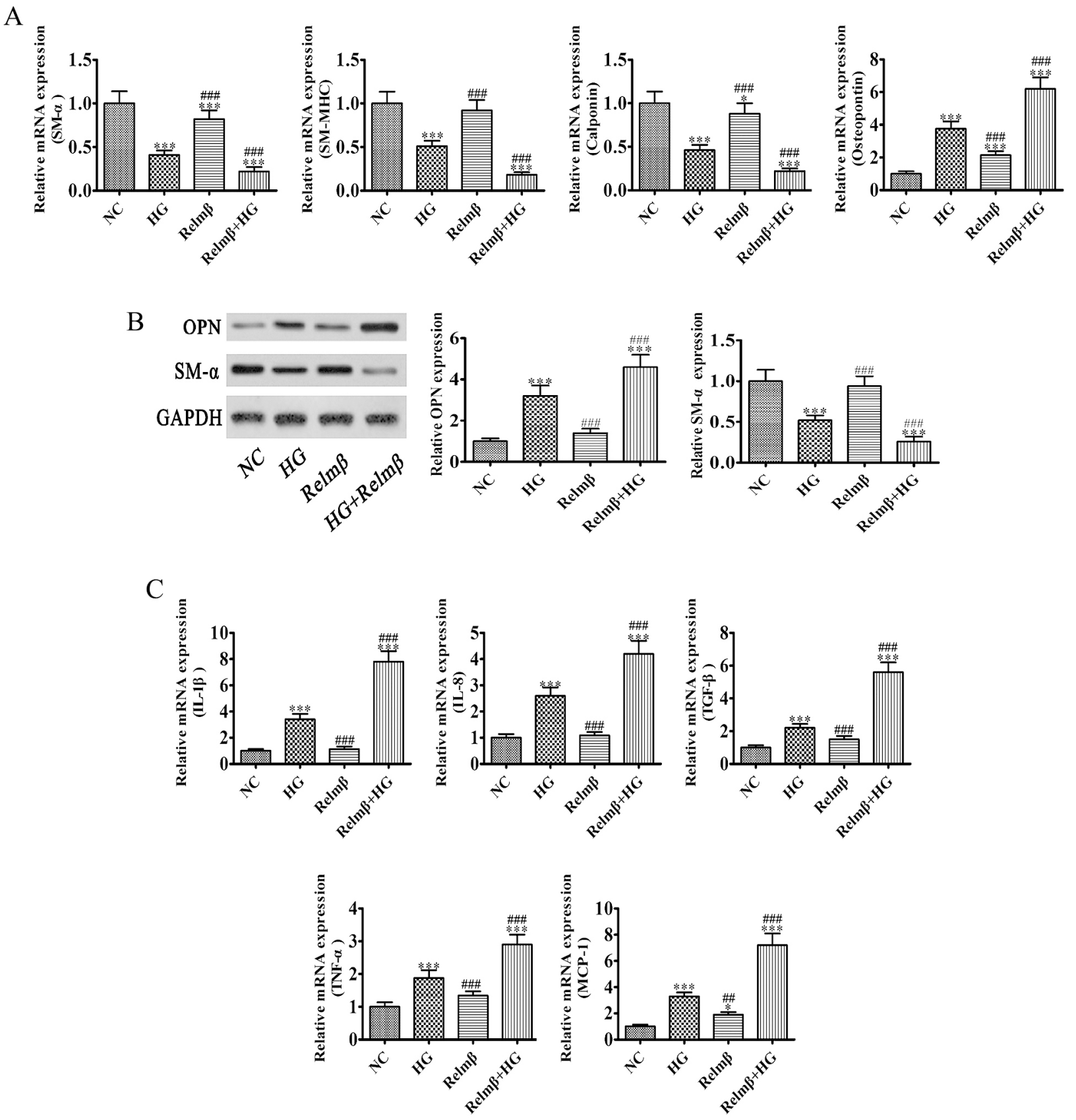

Fig. 1 Relm $\beta$ promotes the phenotypic modulation and inflammation of VSMCs induced by high glucose $(n=3)$. HASMCs were treated with $20 \mathrm{ng} / \mathrm{mL}$ Relm $\beta$ with or without $25 \mathrm{mM}$ high glucose for 24 hours. (A) RT-PCR analyses of smooth muscle $\alpha$-actin ( $\alpha$ SMA), smooth muscle myosin heavy chain (MHC), calponin, matricellular protein, and osteopontin (OPN) in HASMC of four groups. (B) Western blot analyses of $\alpha$-SMA and OPN in HASMC of four groups. Left graph was western blot bands. Right graphs were histogram of quantitative analysis of protein levels $(* * * p<0.001$ compared with control, \#\# $p<0.001$ compared with HG). (C) RT-PCR analyses of inflammation markers including IL-1 $\beta$, IL-8, TGF- $\beta$, TNF $\alpha$, and MCP-1 in HASMCs of four groups. NC, normal control; HG, high glucose. Data are presented as mean \pm SD. $* p<0.05$, $* * * p<0.001$ (compared with NC). \# $p<0.01, \# p<0.001$ (compared with HG).

mesangial cells through p38 signaling [17]. We assessed whether those two pathways are involved in VSMC phenotypic proliferation induced by $\operatorname{Re} \operatorname{lm} \beta$ and high glucose co-stimulation. The expression of p-ERK1/2 and p-p38 was drastically increased by high glucose treatment and was further elevated by $\operatorname{Re} \operatorname{lm} \beta$ and high glucose costimulation (Fig. 4A). After inhibiting p38 and ERK1/2 phosphorylation using p-p38 inhibitor (RWJ64809) and p-ERK1/2 inhibitor (PD98059), the vitality of VSMCs were strikingly attenuated. Moreover, when $\operatorname{Re} \operatorname{lm} \beta$ or high flucose synergistic inhibitor acts on VSCM, the inhibitory effect on cell migration and proliferation are more significant (Fig. 4B, 4C), which suggests that ERK1/2 and p38MAPK signal pathways may be involved 
A
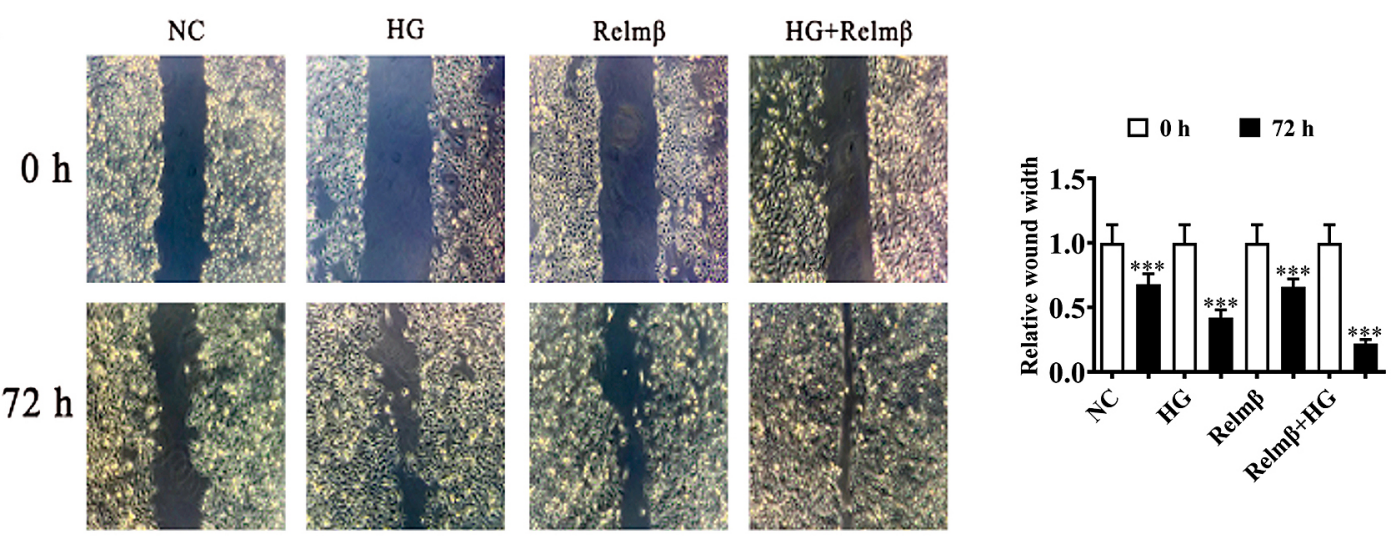

B
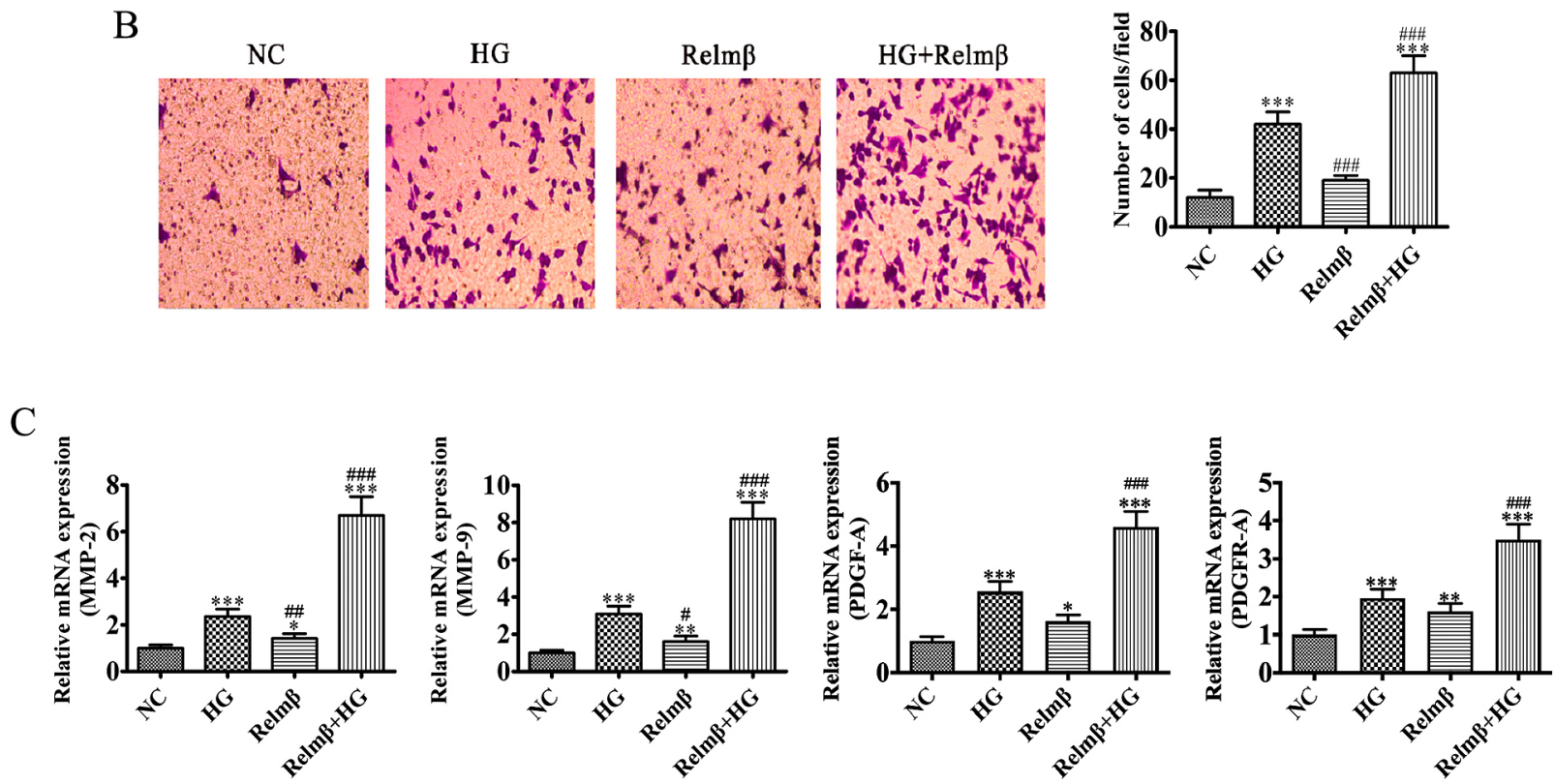

Fig. 2 Relm $\beta$ induces VSMCs migration upon high glucose stimulation $(n=3)$. HASMCs were treated with $20 \mathrm{ng} / \mathrm{mL}$ Relm $\beta$ with or without $25 \mathrm{mM}$ high glucose for 72 hours (A) or 24 hours (B and C). (A) Wound healing assay and (B) Transwell assay in HASMCs of four groups. Left panels were images of wound healing assay and transwell assay, respectively and right panels were histogram of quantitative analysis. (C) RT-PCR analyses of MMP-2, MMP-9, PDGF-A and PDGFR-A in HASMCs of four groups. NC, normal control; NG, high glucose. Data are presented as mean \pm SD. $* p<0.05, * * p<0.01, * * * p<0.001$ (compared with NC). ${ }^{\#} p<0.05,{ }^{\#} p<0.01,{ }^{\# \#} p<0.001$ (compared with HG).

in proliferation of VSMC induced by Relm $\beta$ or high glucose co-stimulation.

\section{Discussion}

VSMCs are highly plastic cells characterized by striking plasticity in response to environmental stimuli [18]. Accumulating evidence shows that VSMC phenotypic plasticity plays an important role in various proliferative vessel disorders, such as essential hypertension and arteriosclerosis [19-21]. However, the details of the molecular mechanisms of VSMC phenotypic modulation are still not elucidated yet. The illustration of the mechanisms regulating the VSMC phenotypic modulation is critical for better understanding the occurrence and development of vascular disorders, especially atherosclerosis. Atherosclerosis always occurs in patients with diabetes and is one of the major causes of diabetes related mortality [22]. High glucose environment may affect VSMC cell cycle and accelerate the development of atherosclerosis, which prompts us to further investigate the regulation of VSMC phenotypic modulation upon high glucose stimulation.

In this study, we investigated the effect of $\operatorname{Re} \operatorname{lm} \beta$ on VSMC in terms of phenotypic modulation, inflammation, migration and proliferation, which are critical steps during atherosclerosis development. Our results were consistent with previous studies that hyperglycemia condition could induce VSMC proliferation and migration $[23,24]$. Additionally, we revealed that $\operatorname{Re} \operatorname{lm} \beta$ could 
A

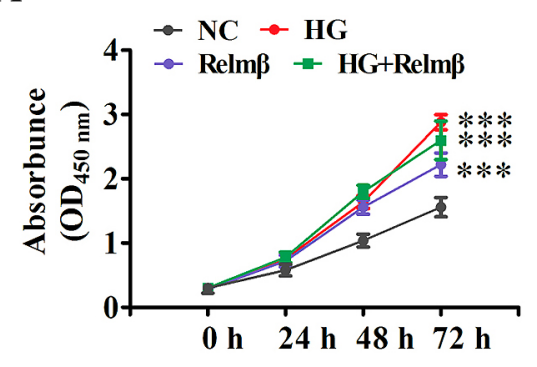

B

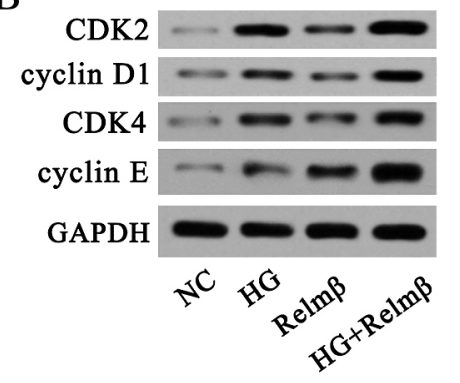

C

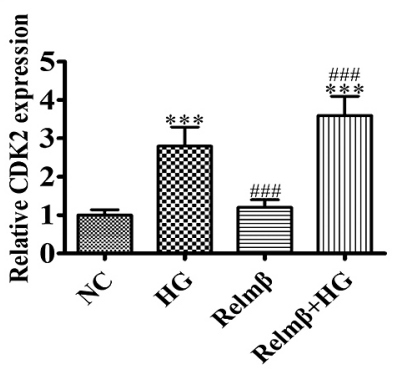

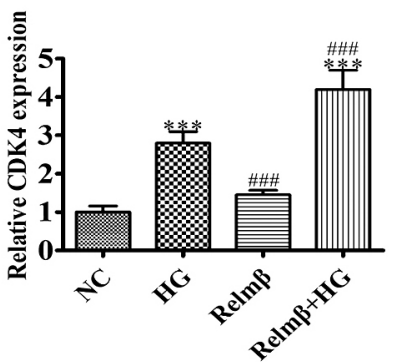
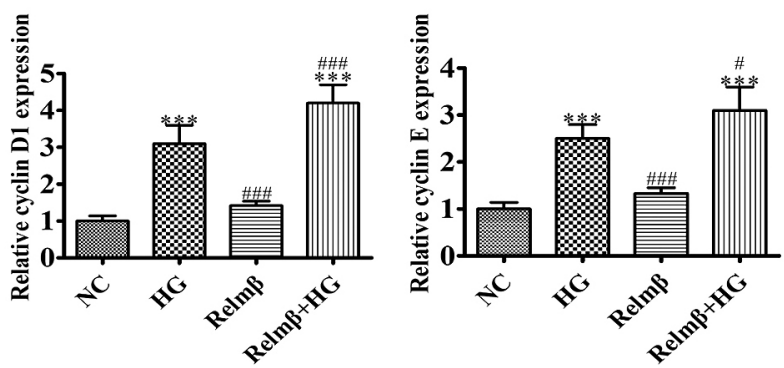

Fig. 3 Relm $\beta$ stimulates proliferation of VSMCs upon high glucose treatment $(n=6)$. HASMCs were treated with 20 ng/mL Relm $\beta$ with or without $25 \mathrm{mM}$ high glucose. NC, normal control; HG, high glucose. (A) CCK-8 assay of HASMCs in four groups. *** $p<$ 0.001 (compared with HG). (B) Cell phase-specific cyclin-dependent kinase (CDK)-cyclins protein expression of HASMCs in four groups. Data are presented as mean $\pm \mathrm{SD}$. *** $p<0.001$ (compared with NC). (C) Histogram of quantitative analysis of protein levels (*** $p<0.001$ compared with control, ${ }^{\#} p<0.05$, ${ }^{\# \#} p<0.001$ compared with HG).

exacerbate the VSMC proliferation and migration induced by high glucose. Unexpectedly, even though comparable levels of absorbunce between HG group and Relm $\beta$ plus HG group were observed when CCK-8 assay was conducted for cell proliferation assessment, the protein levels of CDK2, cyclin D1, CDK4, and cyclin E in HG group were greatly augmented by co-treatment with Relm $\beta$ treatment. This could be because the CCK- 8 assay was so sentitive that high glucose maximized cell proliferation and it's difficult to further be enhanced by Relm $\beta$ treatment.

The mechanisms of the synergistic effect of $\operatorname{Re} \operatorname{lm} \beta$ and high glucose on VSMC is not elucidated yet. Some pathways have been determined to be involved in hyperglycemia including diacylglycerol production, the protein kinase $\mathrm{C}$ pathway activation, MAPKs, advanced glycation accumulation and multiple cytokine production and secretion [25]. MAPKs signaling pathway modulates the expression of numerous transcription factors and participates the VSMC phenotypic modulation [26]. Growing evidence supports that various microRNAs, membrane receptors and ion channels are involved in VSMC phenotypic modulation, migration and proliferation [27]. Whether Relm $\beta$ could synergistically work with these molecules to regulate VSMC phenotype warrant further investigations. Our study also observed that MAPKs pathway, especially ERK1/2 and p38 phosphorylation levels, were significantly induced by $\operatorname{Re} \operatorname{lm} \beta$ and high glucose co-stimulation. More importantly, after suppression of ERK1/2 and p38 pathway by using inhibitors, the proliferation of VSMC was attenuated strikingly. Although the NC groups were also affected by the inhibitor, the more significant down-regulation occurs when high glucose or Relm $\beta$ synergistic inhibitors act in VSCMs. However, the inhibitors also down-regulated the proliferation ability of normal VSCMs to varying degrees, which may indicate that the proliferation effect of high glucose or Relm $\beta$ on cell proliferation was not necessarily caused by P38 or pERK. Whether Relm $\beta$ could synergistically work with these molecules to regulate VSMC phenotype warrant further investigations.

In conclusion, Relm $\beta$ regulates the VSMC phenotypic modulation upon high glucose environment, which could be mediated by MAPKs pathway. These results provide evidence that $\operatorname{Re} \operatorname{lm} \beta$ could be a potential target for the therapeutic strategy of atherosclerosis induced by hyperglycemia.

\section{Highlights}

- $\operatorname{Re} \operatorname{lm} \beta$ as well as high glucose stimulate VSMC phenotypic modulation, migration and proliferation

- Relm $\beta$ augments VSMC phenotypic modulation, migration and proliferation induced by high glucose

- MAPKs pathway are involved in proliferation of VSMC induced by Relm $\beta$ and high glucose co- 
A

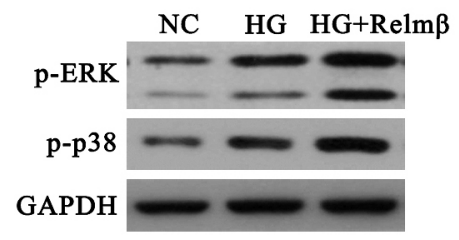

B

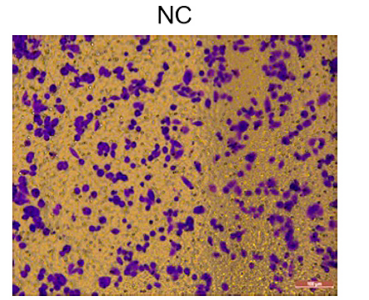

HG

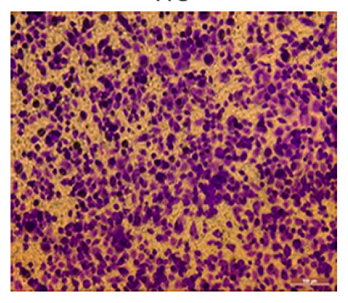

RELM $\beta$

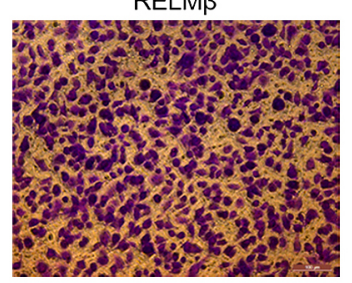

$H G+R E L M \beta$
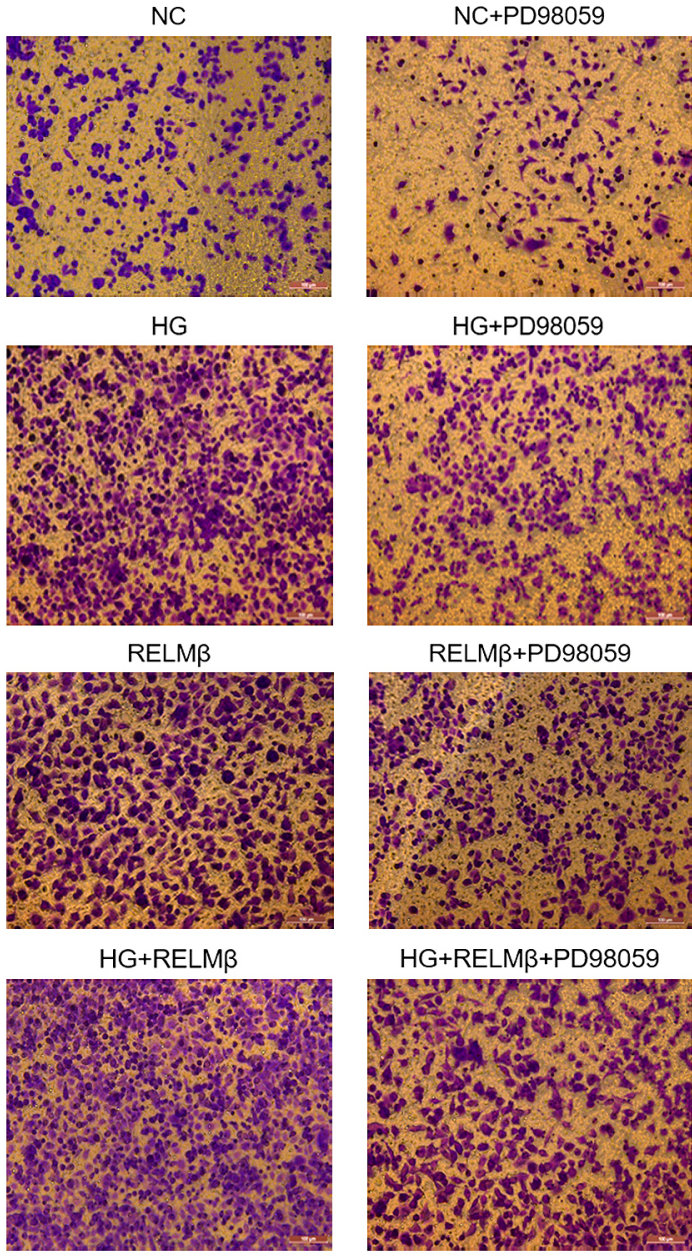

HG+PD98059

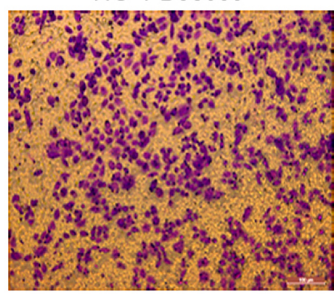

RELMß+PD98059

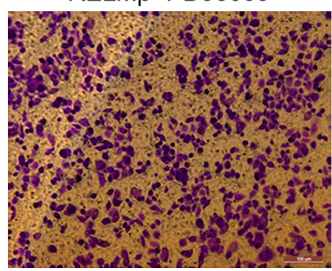

HG+RELM $\beta+P D 98059$

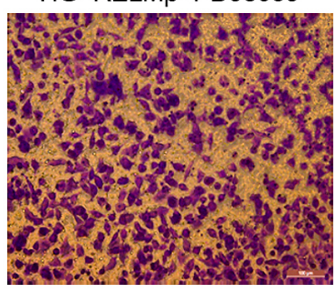

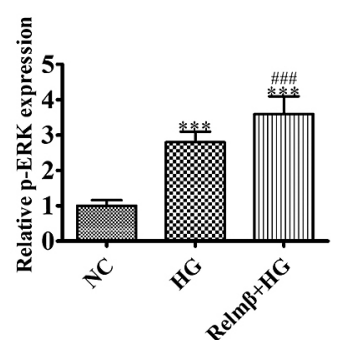

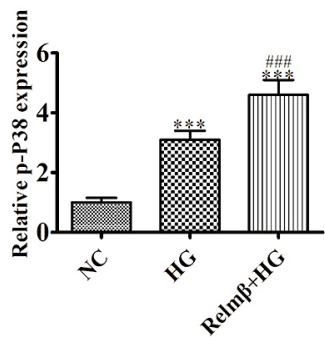

NC+RWJ64809

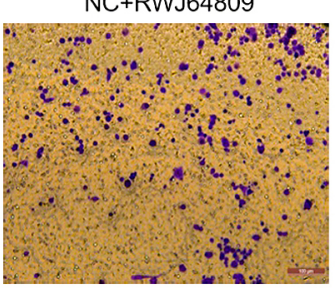

HG+RWJ64809

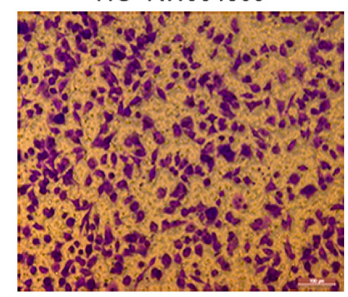

RELM $\beta+R W J 64809$

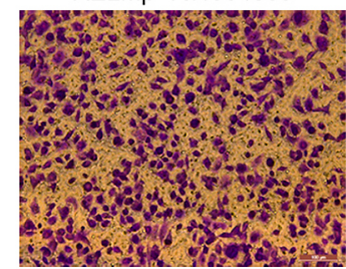

HG+RELM $\beta+R W J 64809$

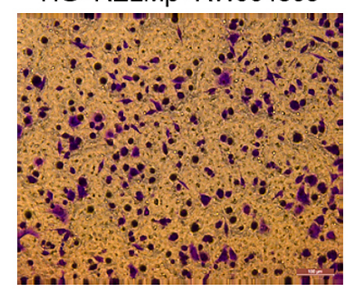

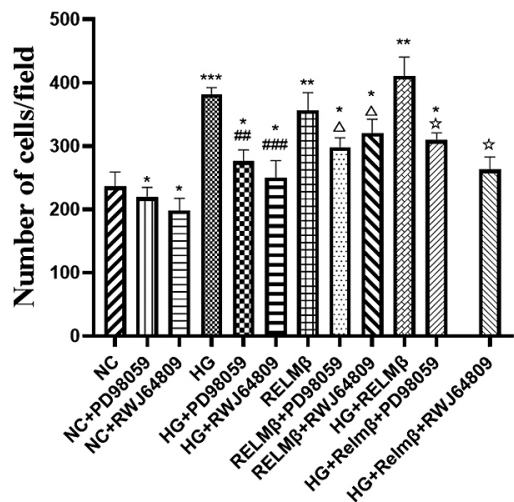

C

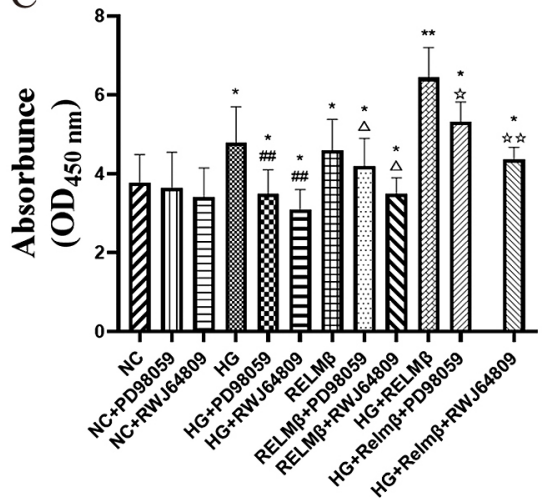

Fig. 4 MAPKs signaling pathway maybe involved in proliferation of VSMCs induced by Relm $\beta$ and high glucose co-stimulation $(n=3)$. (A) Phosphorylation level of ERK1/2 and p38 of in HASMCs treated with high glucose with or without $20 \mathrm{ng} / \mathrm{mL}$ Relm $\beta$ for 24 hours. (B) Representative cell density and (C) CCK-8 of HASMCs after Relm $\beta$ and/or high glucose co-treatment in the absence or presence with p-p38 inhibitor (RWJ64809) $(20 \mathrm{ng} / \mathrm{mL})$ or p-ERK1/2 inhibitor (PD98059) $(20 \mathrm{ng} / \mathrm{mL})(* p<0.05, * * p<0.01$, *** $p<0.001$ compared with control, ${ }^{\#} p<0.01,{ }^{\#} p<0.001$ compared with HG, ${ }^{\Delta} p<0.05$ compared with Relm $\beta$, ${ }^{\text {is }} p<0.05$, 动败 $p<0.01$ compared with $\mathrm{HG}+\operatorname{Re} \operatorname{lm} \beta$ ).

stimulation.

\section{Conflicts of Interest}

The authors declare that there is no conflict of interest.

\section{Acknowledgements}

This work was supported by the National Key R\&D Program of China (2016YFC1305105) and National Natural Science Foundation of China (NO.81670751 and NO.81970711]. 


\section{References}

1. Maranta F, Cianfanelli L, Cianflone D (2020) Glycaemic control and vascular complications in diabetes mellitus type 2. Adv Exp Med Biol 1307: 129-152.

2. Xu H, Jiang J, Chen W, Li W, Chen Z (2019) Vascular macrophages in atherosclerosis. J Immunol Res 2019: 4354786.

3. Shankman LS, Gomez D, Cherepanova OA, Salmon M, Alencar GF, et al. (2015) KLF4-dependent phenotypic modulation of smooth muscle cells has a key role in atherosclerotic plaque pathogenesis. Nat Med 21: 628-637.

4. Hu D, Yin C, Luo S, Habenicht AJR, Mohanta SK (2019) Vascular smooth muscle cells contribute to atherosclerosis immunity. Front Immunol 10: 1101.

5. Shojima N, Ogihara T, Inukai K, Fujishiro M, Sakoda H, et al. (2005) Serum concentrations of resistin-like molecules beta and gamma are elevated in high-fat-fed and obese $\mathrm{db} / \mathrm{db}$ mice, with increased production in the intestinal tract and bone marrow. Diabetologia 48: 984-992.

6. Renigunta A, Hild C, Rose F, Klepetko W, Grimminger F, et al. (2006) Human RELMbeta is a mitogenic factor in lung cells and induced in hypoxia. FEBS Lett 580: 900903.

7. Propheter DC, Chara AL, Harris TA, Ruhn KA, Hooper LV (2017) Resistin-like molecule beta is a bactericidal protein that promotes spatial segregation of the microbiota and the colonic epithelium. Proc Natl Acad Sci USA 114: 11027-11033.

8. Steppan CM, Brown EJ, Wright CM, Bhat S, Banerjee RR, et al. (2001) A family of tissue-specific resistin-like molecules. Proc Natl Acad Sci USA 98: 502-506.

9. He W, Wang ML, Jiang HQ, Steppan CM, Shin ME, et al. (2003) Bacterial colonization leads to the colonic secretion of RELMbeta/FIZZ2, a novel goblet cell-specific protein. Gastroenterol 125: 1388-1397.

10. Vaishnava S, Behrendt CL, Ismail AS, Eckmann L, Hooper LV (2008) Paneth cells directly sense gut commensals and maintain homeostasis at the intestinal hostmicrobial interface. Proc Natl Acad Sci USA 105: 20858 20863.

11. Zheng LD, Tong QS, Weng MX, He J, Lv Q, et al. (2009) Enhanced expression of resistin-like molecule beta in human colon cancer and its clinical significance. Dig Dis Sci 54: 274-281.

12. Rajala MW, Obici S, Scherer PE, Rossetti L (2003) Adipose-derived resistin and gut-derived resistin-like molecule-beta selectively impair insulin action on glucose production. J Clin Invest 111: 225-230.

13. Kushiyama A, Sakoda H, Oue N, Okubo M, Nakatsu Y, et al. (2013) Resistin-like molecule beta is abundantly expressed in foam cells and is involved in atherosclerosis development. Arterioscler Thromb Vasc Biol 33: 19861993.

14. Raines EW, Ferri N (2005) Thematic review series: the immune system and atherogenesis. Cytokines affecting endothelial and smooth muscle cells in vascular disease. $J$ Lipid Res 46: 1081-1092.

15. Ricci C, Ferri N (2015) Naturally occurring PDGF receptor inhibitors with potential anti-atherosclerotic properties. Vascul Pharmacol 70: 1-7.

16. Yu S, Chen Y, Chen S, Ye N, Li Y, et al. (2018) Klotho inhibits proliferation and migration of angiotensin IIinduced vascular smooth muscle cells (VSMCs) by modulating NF-kappaB p65, Akt, and extracellular signal regulated kinase (ERK) signaling activities. Med Sci Monit 24: 4851-4860.

17. Wang YQ, Fan CC, Chen BP, Shi J (2017) Resistin-like molecule beta (RELM-beta) regulates proliferation of human diabetic nephropathy mesangial cells via mitogenactivated protein kinases (MAPK) signaling pathway. Med Sci Monit 23: 3897-3903.

18. Regent A, Ly KH, Lofek S, Clary G, Tamby M, et al. (2016) Proteomic analysis of vascular smooth muscle cells in physiological condition and in pulmonary arterial hypertension: toward contractile versus synthetic phenotypes. Proteomics 16: 2637-2649.

19. Hadrava V, Kruppa U, Russo RC, Lacourciere Y, Tremblay J, et al. (1991) Vascular smooth muscle cell proliferation and its therapeutic modulation in hypertension. Am heart J 122: 1198-1203.

20. Owens GK (2007) Molecular control of vascular smooth muscle cell differentiation and phenotypic plasticity. Novartis Found Symp 283: 174-191; discussion 191-193, 238-241.

21. Shi N, Chen SY (2016) Smooth muscle cell differentiation: model systems, regulatory mechanisms, and vascular diseases. J Cell Physiol 231: 777-787.

22. Shah AD, Langenberg C, Rapsomaniki E, Denaxas S, Pujades-Rodriguez M, et al. (2015) Type 2 diabetes and incidence of cardiovascular diseases: a cohort study in 1.9 million people. Lancet Diabetes Endocrinol 3: 105-113.

23. Milicevic Z, Raz I, Beattie SD, Campaigne BN, Sarwat S, et al. (2008) Natural history of cardiovascular disease in patients with diabetes: role of hyperglycemia. Diabetes care 31 Suppl 2: S155-S160.

24. Li T, Ni L, Liu X, Wang Z, Liu C (2016) High glucose induces the expression of osteopontin in blood vessels in vitro and in vivo. Biochem Biophys Res Commun 480: 201-207.

25. Brownlee M (2005) The pathobiology of diabetic complications: a unifying mechanism. Diabetes 54: 1615-1625.

26. Song SH, Kim K, Jo EK, Kim YW, Kwon JS, et al. (2016) Fibroblast growth factor 12 is a novel regulator of vascular smooth muscle cell plasticity and fate. Arterioscler Thromb Vasc Biol 36: 1928-1936.

27. Zhang MJ, Zhou Y, Chen L, Wang YQ, Wang X, et al. (2016) An overview of potential molecular mechanisms involved in VSMC phenotypic modulation. Histochem Cell Biol 145: 119-130. 